\title{
Strengthening capacity for assessment of HIV-related data needs among key populations to inform evidence-based responses
}

Amrita Rao

Nikita Viswasam

Stefan Baral

Follow this and additional works at: https://knowledgecommons.popcouncil.org/departments_sbsr-hiv

Part of the Demography, Population, and Ecology Commons, Family, Life Course, and Society Commons, Immune System Diseases Commons, International Public Health Commons, and the Medicine and Health Commons

How does access to this work benefit you? Let us know!

\section{Recommended Citation}

Rao, Amrita, Nikita Viswasam, and Stefan Baral. 2020. "Strengthening capacity for assessment of HIVrelated data needs among key populations to inform evidence-based responses," Project SOAR Final Report. Washington, DC: Population Council. 


\section{Strengthening Capacity for Assessment of}

HIV-related Data Needs among Key Populations to Inform Evidence-based Responses

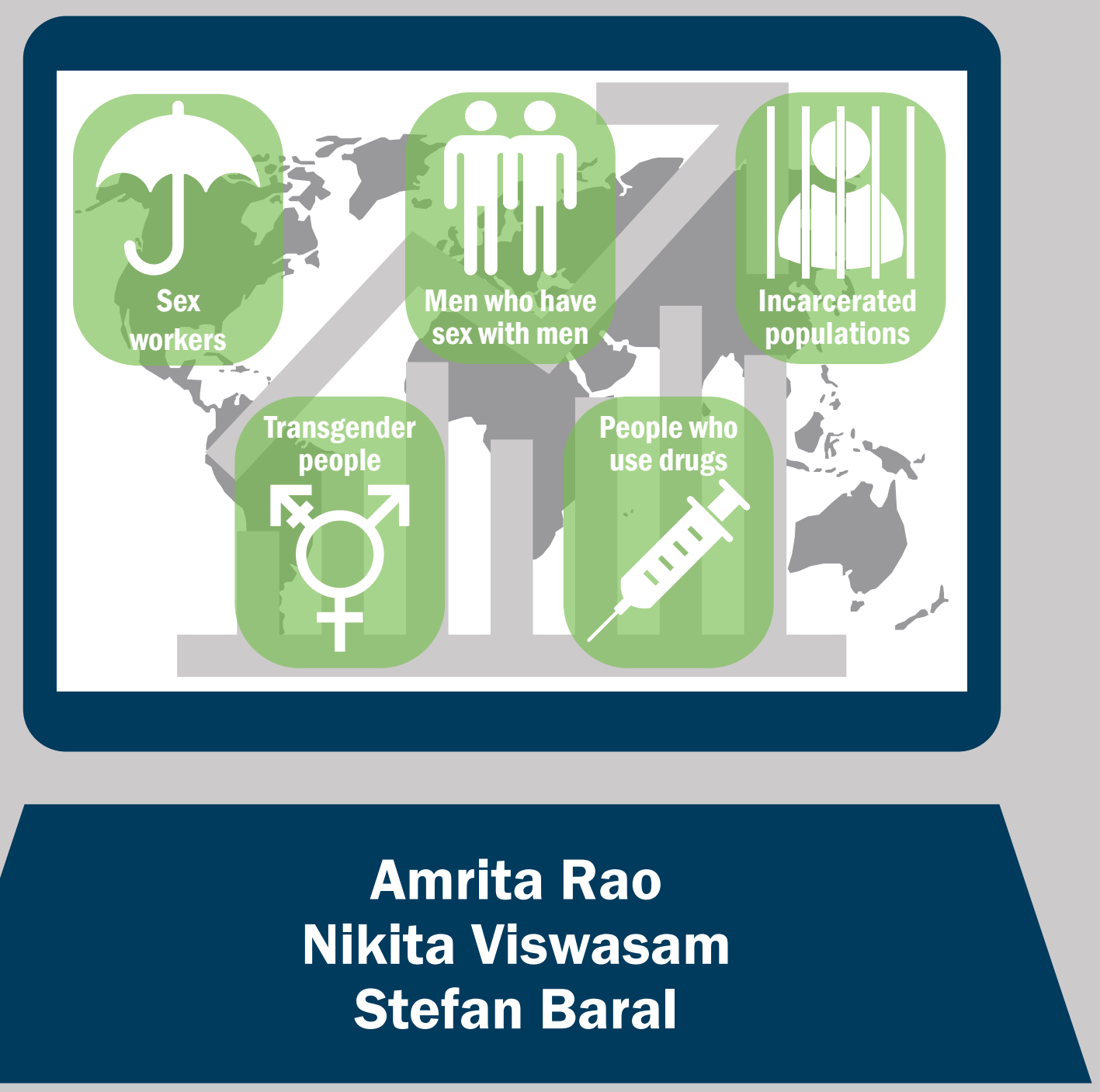


Project SOAR

Population Council

4301 Connecticut Ave, NW, Suite 280

Washington, D.C. 20008 USA

Tel: +1202 2379400

Fax: +1 2022378410

projsoar.org

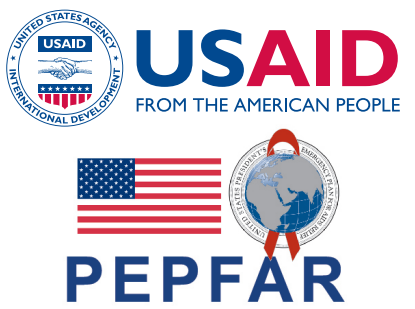

Project SOAR (Cooperative Agreement AID-OAA-A-14-00060) is made possible by the generous support of the American people through the United States President's Emergency Plan for AIDS Relief and the United States Agency for International Development (USAID). The contents of this guidance document are the sole responsibility of Project SOAR and the Population Council and do not necessarily reflect the views of USAID or the United States Government.

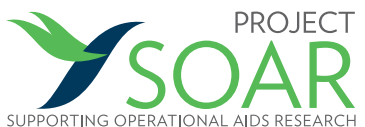

Through operations research, Project SOAR will determine how best to address challenges and gaps that remain in the delivery of HIV and AIDS care and support, treatment, and prevention services. Project SOAR will produce a large, multifaceted body of high-quality evidence to guide the planning and implementation of HIV and AIDS programs and policies. Led by the Population Council, Project SOAR is implemented in collaboration with Avenir Health, Elizabeth Glaser Pediatric AIDS Foundation, Johns Hopkins University, Palladium, and The University of North Carolina.

The Population Council confronts critical health and development issues-from stopping the spread of HIV to improving reproductive health and ensuring that young people lead full and productive lives. Through biomedical, social science and public health research in about 50 countries, the Council works with our partners to deliver solutions that lead to more effective policies, programs, and technologies to improve lives worldwide. Established in 1952 and headquartered in New York, the Council is a nongovernmental, nonprofit organization with an international board of trustees.

The Johns Hopkins Bloomberg School of Public Health has a big mission: Protecting Health, Saving Lives-Millions at a Time. Since its founding in 1916, the Bloomberg School has advanced research, education and practice to create solutions to public health problems around the world. Faculty, staff and students have helped eradicate smallpox, made water safe to drink, improved child survival, reduced the spread of HIV and uncovered the dangers of tobacco smoke. Researchers and scientists are now discovering ways to eliminate malaria, increase healthy behavior, reduce the toll of chronic disease, improve the health of mothers and infants, and change the biology of aging. Every day, the Bloomberg School works to keep millions around the world safe from illness and injury by pioneering new research, deploying knowledge in the field and educating tomorrow's public health leaders.

Published in February 2020. (C2020 The Population Council, Inc.

Suggested citation: Rao, Amrita, Nikita Viswasam, and Stefan Baral. 2020. "Strengthening capacity for assessment of HIVrelated data needs among key populations to inform evidence-based responses," Project SOAR Final Report. Washington, D.C.: Population Council. 


\section{Strengthening Capacity for Assessment of HIV-related Data Needs among Key Populations to Inform Evidence-based Responses}

Amrita Rao, Nikita Viswasam, Stefan Baral

Johns Hopkins University 


\section{ACKNOWLEDGMENTS}

This work was made possible through collaborations with the following individuals and institutions:

Johns Hopkins University

- Epidemiology (Amrita Rao, Nikita Viswasam, Carrie Lyons, Stefan Baral, Global.HIV Group)

- Biostatistics (Abhirup Datta, Tom Louis, Andre Hackman, Andrew Pita)

University of Toronto/Unity Health St. Michael's Hospital

- Sharmistha Mishra, Jesse Knight, Hui Ting Ma

Penn State University

- Le Bao and Maggie Niu

University of North Carolina at Chapel Hill

- Jess Edwards, Sharon Weir, Sabrina Zadrozny

London School of Hygiene and Tropical Medicine

- Elizabeth Fearon, James Hargreaves

PEFPAR/USAID

- Tisha Wheeler, Cameron Wolf, Joseph Lawrence, Alison Cheng, Maria Au, Ashley Frost, Erin Berghammer, Ngaitila Phiri, Lungowe Mwenda-Mwapela, Irum Zaidi, Lisa Nelson

United Nations Joint Programme on HIV/AIDS (UNAIDS)

- Keith Sabin, Mary Mahy, Peter Ghys, Heston Phillips, Nuha Ceesay

Centers for Disease Control and Prevention (CDC)

- Wolfgang Hladik, Helen Savva, Mark Berry, Dimitri Prybylski, Danielle Barradas, Avi Hakim

The Global Fund to Fight AIDS, Tuberculosis and Malaria

- Jinkou Zhao 


\section{TABLE OF CONTENTS}

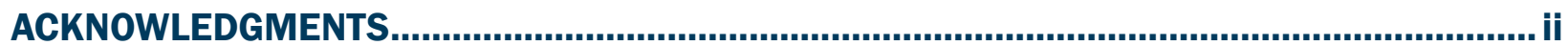

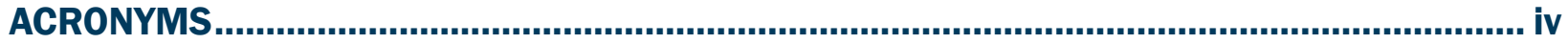

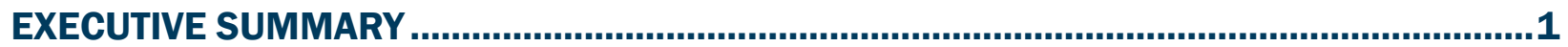

BACKGROUND

SYNTHESIS OF AVAILABLE DATA FOR KEY POPULATIONS ...............................................5

QUALITY ASSESSMENTS AND UTILIZATION OF EPIDEMIOLOGIC DATA............................. 11

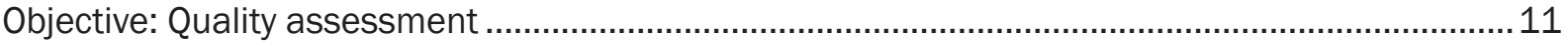

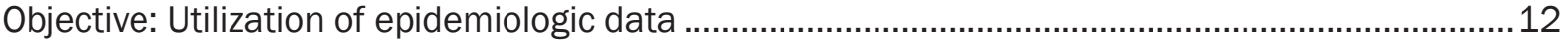

STRENGTHENING CAPACITY IN LINKAGES AND GF PRIORITY COUNTRIES ...................... 14

Objective: Small area estimation .........................................................................................14

Objective: Dynamic transmission modeling .............................................................................18

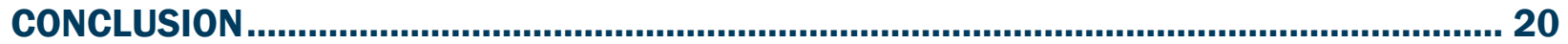

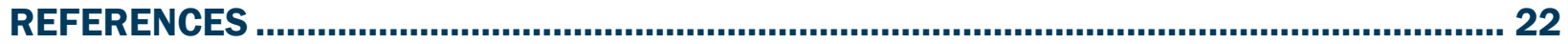

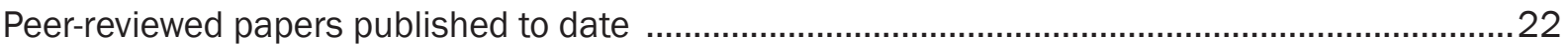

APPENDICES (AVAILABLE AT PROJSOAR.ORG) .............................................................. 23

Appendix A: Key Populations Atlas Advancement Plan

Appendix B: Example data report generated by Global.HIV data repository

Appendix C: Quality Assessment Tool and Scoring

Appendix D: Small area estimation publication

Appendix E: Results of systematic review among FSW and clients

Appendix F: Results of systematic review among MSM

Appendix G: Malawi Workshop Final Report

Appendix H: Eswatini Workshop Final Report

Appendix I: Zambia Workshop Final Report

Appendix J: South Africa Workshop Final Report 


\section{ACRONYMS}

\begin{tabular}{ll} 
ART & Antiretroviral therapy \\
ARV & Antiretroviral drugs \\
BBSS & Biological and Behavioral Surveillance Survey \\
CI & Confidence interval \\
CINAHL & Cumulative Index to Nursing and Allied Health Literature \\
COP & Country operational plan \\
DHS & Demographic and Health Survey \\
FSW & Female sex worker \\
GAM & Global AIDS Monitoring system \\
GF & The Global Fund to Fight AIDS, Tuberculosis and Malaria \\
HIV & Human immunodeficiency virus \\
HIVR4P & HIV Research for Prevention \\
IBBS & Integrated Bio-Behavioral Surveillance Study \\
JHU & Johns Hopkins University \\
KP & Key populations \\
MeSH & Medical subject headings \\
MOT & Modes of transmission models \\
MSM & Men who have sex with men \\
MSW & Male sex worker \\
NGO & Nongovernmental organization \\
OR & Odds ratio \\
PAF & Population attributable fraction \\
PEPFAR & US President's Emergency Plan for AIDS Relief \\
PLACE & Priorities for Local AIDS Control Efforts \\
PrEP & Pre-exposure prophylaxis \\
PRISMA & Preferred Reporting Items for Systematic Reviews and Meta-Analyses \\
PSE & Population size estimates \\
PWUD & People who use drugs \\
SSA & sub-Saharan Africa \\
UNAIDS & United Nations Joint Programme on HIV/AIDS \\
USAID & United States Agency for International Development \\
WHO & World Health Organization \\
\hline M
\end{tabular}




\section{EXECUTIVE SUMMARY}

Defining the HIV prevention and treatment needs for key populations, who have disproportionate HIV acquisition and transmission risks, has been particularly difficult in the context of generalized HIV epidemics where less attention has historically been placed on the HIV prevention and treatment needs of these groups. There is a gap in our understanding of the specific needs of-and ultimately the investment case for the added value of supporting-disproportionately burdened key populations in these settings. In response to this gap, Johns Hopkins University under the United States Agency for International Development (USAID)-funded Project SOAR implemented a project in partnership with collaborators, with two primary purposes: (1) synthesizing and assessing the quality of available data for key populations; and (2) leveraging these data to strengthen capacity of a strategic group of governmental, non-governmental, and community stakeholders to effectively use these data to prioritize rights-based, comprehensive data collection efforts and programmatic responses. The specific activities, objectives, and key results included:

\section{SYNTHESIS OF AVAILABLE DATA FOR KEY POPULATIONS}

\section{Objectives}

- Complete a global systematic review of all available data among key populations characterizing the burden of HIV, the HIV treatment cascade, key prevention indicators, population size estimates (PSEs), and selected structural determinants of HIV.

- Build a data repository housing all available data gathered from the systematic review.

- Methods: A systematic review of studies and reports published or made available between 2006 and 2019 was conducted to capture various measures to assess the burden and risk of HIV. Based on defined inclusion and exclusion criteria and a review team analysis, data were abstracted from 1,059 studies for female sex workers (FSWs), 1,186 studies for men who have sex with men (MSM), 251 studies for transgender women, 292 studies for incarcerated populations, and 1,351 studies for people who use drugs.

- Results: Abstracted data, including key PSEs, are now in the process of being incorporated into the UNAIDS Key Populations Atlas. 


\section{QUALITY ASSESSMENTS AND UTILIZATION OF EPIDEMIOLOGIC DATA}

\section{Objective}

- Conduct quality assessments on a subset of HIV data on key populations from 30 priority countries using an adapted quality assessment tool for assessing key populations' data.

- Methods: A quality assessment tool for key populations research was developed, adapted, and applied to available data points for 30 priority countries.

- Results: Quality assessments are now housed in the data repository and have been shared with in-country stakeholders during capacity-building workshops.

\section{Objective}

- Systematically assess the level of utilization of epidemiological data in HIV policy and program documents across Africa, using population size estimation identified through the Global.HIV data repository.

- Methods: Twenty-three different types of documents released between 2009 and 2019 used to inform HIV programming in countries, with a focus on PEPFAR and the Global Fund to Fight AIDS, Tuberculosis and Malaria (GF) investments, were reviewed.

- Results: The review found that of 120 PSEs identified, a total of 24 were referenced in official policies. The review showed that there is a strong need to build capacity to interpret the quality of data in terms of understanding strengths and weaknesses of the methods used to derive estimates.

\section{STRENGTHENING CAPACITY IN LINKAGES AND GF PRIORITY COUNTRIES}

\section{Objective}

- Conduct small area estimation, an evidence-based approach to address data gaps in the HIV response, for six priority countries.

- Methods: For each estimation, a linking model was developed using existing direct estimates and relevant auxiliary data, and an exploratory spatial analysis was utilized to establish point patterns of data to detect potential clusters of communities.

- Results: Small area estimation techniques were tailored to specific populations (e.g., MSM, FSW) and drew upon a variety of selected and appropriate data sources. Specific findings are included in appendices. 


\section{Objective}

- Conduct Dynamic Transmission Modeling exercise to rigorously evaluate, through systematic reviews and generic modeling, the contribution of FSWs and their clients and MSM in diverse HIV epidemic contexts across sub-Saharan Africa (SSA).

- Methods: An independent systematic review was conducted, odds ratios of prevalent HIV for FSWs and MSM were compared to the general population, and dynamic models of HIV transmission were used to explore the utility of the classic population attributable factor.

- Results: The FSW review estimated an HIV prevalence in FSWs and clients of 28 percent and 6 percent, respectively, and an incidence rate ranging from 0.4 percent to 71 percent and 0 to 88 percent, respectively. Relative HIV expenditure on sex work programs was 0-3 percent. The MSM review found an estimated prevalence of HIV diagnosis and awareness among MSM living with HIV in SSA of 27 percent.

At the conclusion of this activity a series of novel and innovative resources became available for local and global stakeholders. An extensive global systematic review that captures the burden of HIV for key populations and their engagement in the treatment cascade has been completed and is now being integrated into the KP Atlas, a collaborative effort with USAID that will yield a user-friendly data platform and website that displays HIV status for key populations in more than 150 countries. Quality assessments were conducted on available data for 30 priority countries based on an adapted assessment, and results of the quality assessments have been shared with participants at capacity strengthening workshops and will eventually be accessible as part of the KP Atlas. An assessment of the utilization of epidemiologic data was also conducted and showed that only a small proportion of size estimation studies are being used to inform programming within country governments and with their funding and implementation partners. This finding underscores the importance of improving methods and tools to ensure the effective uptake of epidemiological data, including size estimation data.

Capacity-building workshops were conducted in six countries to highlight existing data and promote better understanding of epidemiologic data and its application to policy development. In several instances, small area size estimation and dynamic modeling were conducted to further strengthen the utility of available data. The work reported here has also informed country operational plan 2019 activities at both a headquarters and local level. 


\section{BACKGROUND}

Knowing and understanding HIV acquisition risks in specific contexts and settings is key to informing an effective response, as detailed in the Global Fund to Fight AIDS, Tuberculosis and Malaria (GF) 2017-2021 strategy and PEPFAR 3.0. Defining the HIV prevention and treatment needs for key populations, however, who have disproportionate HIV acquisition and transmission risks, has been particularly difficult in the context of generalized HIV epidemics where less attention has historically been placed on the HIV prevention and treatment needs of these groups. Where data are available, and have been effectively analyzed, mathematical models have supported the need for specific HIV prevention and treatment interventions for key populations across epidemic settings.

The response to preventing and treating HIV has differed from most other clinical conditions in that the implementation of preventive measures is generally managed by civil society and non-profit, non-governmental institutions rather than health care facilities. While significant investments in epidemiologic and intervention studies for key populations have been made, they may not be fully realized given a lack of capacity to assess data quality and meaningfully apply collected data to decision-making about large-scale HIV funding and programmatic responses. Data may also not be readily accessible. For example, data such as population size estimation studies for key populations, that can be combined with data from integrated bio-behavioral surveillance (IBBS) studies to inform program structure and content, holds real promise for the development of evidence-based programs. Dedicating resources to ensuring that all potentially informative data are available and accessible and that stakeholders in high impact areas have the capacity to assess and apply these data is critical.

In response to gaps in both defining the burden and need of key populations in generalized epidemics and utilizing quality data about burden to inform an effective response, the project was implemented under the United States Agency for International Development (USAID)-funded Project SOAR by Johns Hopkins University, in partnership with collaborators, with two primary purposes: (1) synthesizing and assessing the quality of available data for key populations; and (2) utilizing the findings to strengthen capacity of a strategic group of governmental, nongovernmental, and community stakeholders to effectively use these data to prioritize rightsbased, comprehensive data collection efforts and programmatic responses. 


\section{SYNTHESIS OF AVAILABLE DATA FOR KEY POPULATIONS}

\section{Objectives}

- Complete a global systematic review of all available data characterizing the burden of HIV, the HIV treatment cascade, key prevention indicators, population size estimates (PSEs), and selected structural determinants of HIV, among key populations.

- Build a data repository housing all available data gathered from the systematic review.

\section{Information sources and search strategies}

A systematic review of studies and reports published or made available between 1 January 2006 and 1 January 2019, was conducted to capture the burden and risk of HIV, including prevalence and incidence estimates, prevention indicators and treatment cascades, PSEs, experienced violence, and engagement with healthcare systems. This protocol is registered in the PROPSERO database (CRD42016047259; 28 September 2016) and is in accordance with the guidelines specified in the Preferred Reporting Items for Systematic Reviews and Meta-analyses statement for protocols (Moher et al. 2009).

The following databases were searched for studies and reports: PubMed $^{\odot}$, EMBASE ${ }^{\odot}$, Global Health ${ }^{\odot}$, SCOPUS $^{\odot}$, PsycINFO $^{\odot}$, Sociological Abstracts ${ }^{\odot}$, CINAHL $^{\odot}$ (Cumulative Index to Nursing and Allied Health Literature), Web of Science ${ }^{\odot}$, and POPLine ${ }^{\odot}$. Peer-reviewed conference abstracts were searched from online publications of conference proceedings, including those from the International AIDS Conference; the Conference on HIV Pathogenesis, Treatment, and Prevention; HIV Research for Prevention (HIVR4P); and the Conference on Retroviruses and Opportunistic Infections (CROI). The World Health Organization (WHO) publications database was also searched as well as the National Library of Medicine's Meeting Abstracts database (NICHSR online). Other data sources from the gray literature were identified through the USAID's Development Experience Clearinghouse (USAID DEC online), including national surveillance system data reports such as the Demographic and Health Surveys and Integrated Biological and Behavioral Surveillance Surveys, as well as studies conducted by large international non-governmental organizations.

Search strategies were developed based on a combination of controlled vocabulary (e.g., MeSH terms) and other keyword searches and were adapted fromexisting search strategies developed for earlier systematic reviews of key populations conducted by our team. Multiple iterations of the search strategies were piloted in order to prioritize a highly sensitive search that captured all relevant available data. The search was determined to be highly sensitive by testing that confirmed that the search returned all expected articles along with others that may be less relevant but still important to review. Search strategies comprised search terms for three independent concepts: terms for the population of interest; HIV-related terms, and terms related 
to violence. Each search run was a combination of concept one AND concept two OR concept three.

\section{Inclusion and exclusion criteria}

To be included in the review, studies met the following criteria:

1. Studies of any design that include either HIV data or violence data (see Table 2 for all captured indicators) among female sex workers (FSWs), men who have sex with men (MSM), people who use drugs (PWUD), transgender populations, and incarcerated populations, even if these groups were not the main focus of the study.

2. Participants in studies were of any age, race, or ethnicity.

3. Studies were published in a peer-reviewed journal, presented as an abstract at a scientific conference, or available on the web from governmental or non-governmental sources.

4. Qualitative studies and modeling studies were included as searchable records in the data repository, but qualitative data were not abstracted or included in the quantitative narrative analysis.

5. Published or presented between 1 January 2006 and 1 January 2019.

6. Data from all countries and settings were included.

The following types of studies were excluded from the review:

1. Studies where the sample size was less than 50.

2. Studies published in languages other than English, French, or Spanish.

\section{Screening and selection}

Titles, abstracts, citation information, and descriptor terms of citations identified through the search strategy were screened by a team of reviewers and were selected to move to full-text review if the above six criteria were met. Full-text articles were obtained of all selected abstracts and two independent reviews were conducted of each article to assess all full-text articles for eligibility for final study selection. The same set of questions were used for full-text screening. Title/abstract and full-text review were conducted in Covidence $^{\odot}$, a tool designed to help facilitate the systematic review process, produced in partnership with Cochrane Reviews (Covidence online). Differences were resolved by a third independent reviewer.

In total, data were abstracted from 1,059 studies for FSWs, 1,186 studies for MSM, 251 studies for transgender women, 292 studies for incarcerated populations, and 1,351 studies for PWUD. Below is a summary table that displays the geographic distribution of studies identified and abstracted: 
Table 1 Geographic distribution of studies identified and abstracted for systematic review

\begin{tabular}{|lc|}
\hline World region & Percent of total library records \\
Asia & 29 \\
Central America, South America, Caribbean & 8 \\
Sub-Saharan Africa & 13 \\
North Africa and Middle East & 3 \\
North America & 31 \\
Europe & 9 \\
Oceania & 3 \\
Worldwide & 3 \\
\hline
\end{tabular}

\section{Data abstraction}

Data were abstracted independently by a team of reviewers using standardized data abstraction forms in REDCap. Differences in data abstraction were resolved using REDCap's data comparison tool by a third, independent reviewer. Reviewers were trained on how to abstract available information from eligible articles and how to index the article in the database. Reviewers noted all available data captured in the REDCap tool (Table 2). The following information was gathered from each included study: study identification including author(s); citation; year of publication and study description including location, setting, and population; years (period of study); study design; sample size; age range; sex; and gender, if reported separately. 
Table 2 Data fields captured in the REDCap Global.HIV data repository

Indicator/Fields

1. Unique ID

2. Web link

3. Title

4. First name

5. Last name

6. Year of publication

7. Cohort ID

8. Journal name

9. Primary focus on quantitative data

10. Type of study/document

11. Study recruitment method

12. Region of interest

13. Number of countries represented in article

14. Number of populations of interest

15. Article/report contains data of male sex workers (MSW) $(\mathrm{Y} / \mathrm{N})$

16. Sample size

17. Prevalence of HIV in sample (count)

18. Incidence of HIV in sample (count)

19. Number with knowledge of HIV prevention

20. Number who report condom availability

21. Number who report PrEP availability

\section{Definition}

Unique ID generated to identify each article using a combination of author last name, journal, title, and publication year

Web link where article is found

Title of the article being abstracted

First name of the first author

Last name of the first author

Year of publication

ID generated to identify papers published from the same study/ cohort

Full name of journal where article was published

Based on the project design and methods, provide information on primary focus of data.

Cross sectional, longitudinal, randomized controlled trial, other

Based on above selection, provide information on sample size, year/duration, and recruitment method

Global region of data collection

Countries for which data was reported

Key populations for which data was reported

Availability of data on male sex workers, particularly in articles that report MSM data

Total sample may contain key population of interest as well as those not of interest.

Number of HIV-positive individuals in the sample

Number of new cases of HIV infections in the sample (not previously diagnosed)

Study measured and reported HIV knowledge in sample

Study included measures on availability of condoms as reported by participants

Study included measures on availability of PrEP (pre-exposure prophylaxis) as reported by participants 
22. Number tested (ever)

23. Number tested in last 12 months

24. Number previously diagnosed

25. Number linked to HIV care

26. Number retained in care

27. Number on treatment

28. Number adherent/ undetectable

29. Size estimate
Study measured number of participants who report ever taking an HIV test in their lifetime

Study measured number of participants who report taking an HIV test in the 12 months prior to the study

Participants who report having been diagnosed with HIV before the study (self-report being HIV positive)

Participants or study report linkage to HIV care

Participants report being retained in HIV care

Participants report being on HIV treatment (ART, ARV)

Participants who report being adherent to treatment, or report having an undetectable viral load (i.e., taking treatment regularly makes their viral load undetectable)

Study reports on estimating the size of the key population by different regions. Known as population size estimation, population size estimate, or size estimate. Study will report different methods of estimation. (Fields available for up to 4 size estimates.)

30. Size estimate, lower bound of Upper and lower bounds of estimate confidence interval $\mathrm{Cl}$ Upper bound of $\mathrm{Cl}$

31. Specific region for size estimate

Provide region within country or country for which estimate was made.

Reported method of size estimate conducted (Ex: capturerecapture, wisdom of the crowds, unique object distribution, mapping and enumeration)

33. Number experienced physical violence

Participants who report physical violence (not sexual or intimate partner violence)

Participants who report sexual violence

34. Number experienced sexual violence

35. Number experienced intimate partner violence

Participants who report intimate partner violence

36. Number who have consistent condom use

Participants report using condoms consistently (sometimes measured as "always use condoms" as compared to "sometimes" or "never"). Some articles may report consistent condom use for both receptive and insertive anal sex.

37. Number denied health services

Participants who report having been denied health care from health service providers

Participants who report being afraid to seek health services (due to stigma, discrimination, or past negative experiences with health providers) 
Collectively, the systematic review identified 120 population size estimates (PSEs) from 41 studies for key populations from the 48 sub-Saharan African countries, which were later included in a research utilization assessment discussed on page 12. Overall, 73 PSEs were available for FSWs, 28 for MSM, 21 for PWUD, and none for transgender. Either national, regional, or local PSEs were available from 22 different countries including Angola, Burkina Faso, Burundi, Cameroon, Cote d'Ivoire, Egypt, Eswatini, Ethiopia, Ghana, the Gambia, Kenya, Mauritius, Mozambique, Morocco, Niger, Nigeria, Rwanda, Senegal, South Africa, Tanzania, and Togo. Three regional estimates were available, two of which were for Eastern Africa. Overall, 73 of the PSEs were available in peer reviewed literature, and 48 were from gray literature.

\section{Repository development and integration with KP Atlas}

Given the utility of the data repository, it has been decided that it will be integrated into the UNAIDS Key Populations (KP) Atlas with the aim of developing a user friendly, interactive website that displays HIV statistics for key populations for more than 150 countries. In addition to data from UNAIDS and the Johns Hopkins University (JHU)/Project SOAR repository, data from the CDC and the Global Fund will be included. Currently, the KP Atlas displays HIV indicators data and surveillance reports sent annually by country offices to UNAIDS through their Global AIDS Monitoring system. A newly integrated KP Atlas Data will provide a global map interface that will show both overall HIV prevalence data as well as country-level key population data as generated through the global systematic review. In addition, the tool will support comparisons between different countries and between different sub-national units within a country. The KP Atlas platform will also provide country dashboard pages, downloadable datasets tailored to user needs, and story maps that weave together contextual information, key data, anecdotal evidence, and photos and figures. The full KP Atlas Advancement Plan is found in Appendix A.

\section{Repository access}

While work proceeds on integrating the repository with the KP Atlas, individuals and organizations are able to access the repository. A few users, who have been collaborating on the KP Atlas merging process and in ongoing work on small area estimation and dynamic transmission modeling, have direct access to the database; for others, data pull requests can be made through a Google form. To date, data reports generated by the repository have been requested and produced for FHI360, Population Council, UNAIDS, UNAIDS Modeling Reference Group, USAID Headquarters, and the WHO, among other organizations, the data from which has informed the UNAIDS World AIDS Day report and the Country Operation Planning in Southern Africa, among other activities. An example of a requested data report generated by the REDCap data repository for a client organization is available in Appendix B. 


\section{QUALITY ASSESSMENTS AND UTILIZATION OF EPIDEMIOLOGIC DATA}

\section{OBJECTIVE: QUALITY ASSESSMENT}

Conduct quality assessments on a subset of HIV data on key populations from 30 priority countries selected based on current HIV programming priorities, prevalence, incidence, treatment cascades, and PSEs using a quality assessment tool developed for assessing key populations data.

\section{Developing a quality assessment tool}

A quality assessment tool for key populations research was developed and adapted from the NHLBI Quality Assessment Tool for Observational Cohort and Cross-Sectional Studies (NHLBI online). The tool was adapted by the study team in order to capture the essential elements of the original tool, while also ensuring applicability to relevant outcomes. The tool was designed such that two independent assessors evaluated first the general study design and the study implementation and then evaluated indicator-specific data quality. The quality assessment tool was designed to assess the quality of available evidence for data points of HIV prevalence, incidence, the HIV care continuum, and PSE. Datapoints were categorized as "good," "fair," or "poor" based on an evaluation of a number of criteria related to study design, study implementation, and use of appropriate analytic methods. All articles available for a specific country were gathered and efforts were taken to group publications based on the study of origin. Papers from the same original study were reviewed together to evaluate the quality of the reported data from the single study. The quality assessment tool is available in Appendix $\mathrm{C}$. 
The quality assessment tool was applied to available data points for 30 priority countries. Countries were selected from the larger review after discussions with partners and stakeholders and based on current donor priorities-where resources are invested, where current programs for key populations and HIV exist, and where more data are needed to inform programs. Selected countries included Angola, Botswana, Burundi, Cambodia, Cameroon, Cote d'Ivoire, Democratic Republic of Congo, Dominican Republic, Ethiopia, Ghana, Haiti, India, Indonesia, Kenya, Lesotho, Mozambique, Myanmar, Namibia, Nigeria, Papua New Guinea, Rwanda, South Africa, South Sudan, Tanzania, Uganda, Ukraine, Vietnam, Zambia, and Zimbabwe.

\section{Results}

Results of the quality assessments are also housed in the REDCap data repository and can be accessed upon request. Quality assessments have been shared with in-country stakeholders during the capacity-building workshops discussed below. During each capacity-building workshop, the tool's utility is reviewed as is the underlying methodology used in its development and results from a review of in-country data. In these workshops, decision-makers learn how to use the quality assessment to both differentiate among presented data but also how to utilize the quality assessment tool in the future to be able to conduct quality assessments of their own.

\section{OBJECTIVE: UTILIZATION OF EPIDEMIOLOGIC DATA}

Systematically assess the level of utilization of epidemiological data in HIV policy and program documents across Africa, including sub-Saharan and North Africa, using PSEs identified through the Global.HIV data repository.

\section{Assessing utilization}

We reviewed 23 different types of documents released between 2009 and 2019 used to inform HIV programming in countries, with a focus on PEPFAR and the GF investments. We focused on PSEs in particular as PSEs are necessary for informing interventions for key populations, service target development, and contextualizing HIV service access. Utilization review included an assessment of whether available PSE data were identified and referenced to inform policy, programming, or resource allocation. Policy and program documents were evaluated using a series of Research Utilization Indicators, as well as Stakeholder Engagement Indicators listed below. 


\begin{tabular}{|c|c|}
\hline Research utilization indicators & Stakeholder engagement indicators \\
\hline $\begin{array}{l}\text { Have stakeholders developed an interpretation } \\
\text { and use plan for size estimation data? }\end{array}$ & $\begin{array}{l}\text { Have stakeholders been identified who would } \\
\text { be needed to make policy/program decisions? }\end{array}$ \\
\hline $\begin{array}{l}\text { Have size estimation data been used to identify a } \\
\text { problem? }\end{array}$ & $\begin{array}{l}\text { Have stakeholders been identified who } \\
\text { represent the target population? }\end{array}$ \\
\hline $\begin{array}{l}\text { Have size estimation data been used to develop } \\
\text { a plan of action/recommendation to address that } \\
\text { problem? }\end{array}$ & $\begin{array}{l}\text { Have stakeholders been engaged throughout } \\
\text { study design? }\end{array}$ \\
\hline $\begin{array}{l}\text { Have size estimation data been used to direct } \\
\text { service delivery? }\end{array}$ & $\begin{array}{l}\text { Have stakeholders been engaged throughout } \\
\text { study implementation? }\end{array}$ \\
\hline $\begin{array}{l}\text { Have size estimation data been used to change a } \\
\text { GF program or policy as documented in concept } \\
\text { notes? }\end{array}$ & $\begin{array}{l}\text { Were study data/results shared by } \\
\text { stakeholders with other groups? }\end{array}$ \\
\hline $\begin{array}{l}\text { Have size estimation data been used to change } \\
\text { a PEPFAR program or policy as documented in } \\
\text { country operational plans (COPs)? }\end{array}$ & $\begin{array}{l}\text { Were stakeholders included as authors on } \\
\text { published document? }\end{array}$ \\
\hline $\begin{array}{l}\text { Have size estimation data been used to change a } \\
\text { national MOH program or policy as documented in } \\
\text { national strategic plans? }\end{array}$ & $\begin{array}{l}\text { Was a study-specific advisory panel } \\
\text { established? }\end{array}$ \\
\hline $\begin{array}{l}\text { Results/data translated into non-academic } \\
\text { resources (briefs/advocacy tools) }\end{array}$ & \\
\hline
\end{tabular}

\section{Results}

The review found that of the 120 PSEs identified, two PSEs were referenced in a change to GF program or policy, 14 PSEs were referenced in a change to PEPFAR program or policy as documented in the COPs, and 8 PSEs were referenced in a change to a national ministry's of health program or policy as documented in national strategic plans. Of the 59 PSEs identified from the peer-reviewed literature, 17 were translated into non-academic resources, including briefs, pamphlets, or advocacy tools. There are several reasons that existing PSEs may not have been fully utilized. In some instances, there may not be political will to address the needs of key populations and in turn consider relevant PSE data. There also appears to be limited capacity to understand the relative quality of available estimates. Finally, given that estimates are often missing in many geographic areas, existing PSEs are often discounted as not being of relevance to the program (i.e., only conducted in one city and not generalizable). Our review showed that there is a strong need to build capacity to interpret the quality of data in terms of understanding strengths and weaknesses of the methods used to derive estimates. 


\section{STRENGTHENING CAPACITY IN LINKAGES AND GF PRIORITY COUNTRIES}

In-country workshops were conducted in LINKAGES and GF priority countries to highlight existing data as well as gaps in knowledge, and promote better understanding of epidemiologic data and the application to policy development. Six countries (Cameroon, Cote d'Ivoire, Eswatini, Malawi, South Africa, and Zambia) were selected based on conversations with LINKAGES, the Global Fund, USAID, and others to determine where these capacity-building workshops and more indepth work would be useful. To maximize the benefit of these workshops, a series of small area estimation analyses were conducted as well as some targeted dynamic transmission modeling as discussed below. Reports for each of the in-country capacity strengthening workshops, including a second in-person capacity strengthening workshop which was conducted in Malawi are included in Appendices $\mathrm{H}-\mathrm{K}$.

\section{OBJECTIVE: SMALL AREA ESTIMATION}

Conduct small area estimation, an evidence-based approach to address data gaps in the HIV response, for six priority countries.

\section{Small area estimation in support of capacity-strengthening workshops}

Small area estimation of key population size was conducted for six LINKAGES and GF countries (Cameroon, Cote d'Ivoire, Eswatini, Malawi, South Africa, and Zambia). Results of these analyses were presented at capacity-building workshops to provide key stakeholders with feedback on the methods and results. Small area estimation is a set of statistical techniques that makes use of available data to predict a parameter, in this case population size, where data do not exist. PSEs are central to defining the necessary scale of specific programs and program targets. Prediction and precision of subnational denominators and PSEs can be improved using small area estimation and advanced extrapolation methods, including Bayesian approaches to better account for uncertainty at different levels of aggregation. A linking model is developed to predict indirect estimates using existing direct estimates and relevant auxiliary data. Possible auxiliary data sources include key social and economic indicators; routinely-collected surveillance data and information about health service provision; social media search data, including Facebook and Google search data; and other relevant data sources that can serve to improve prediction models.

Exploratory spatial analysis can be utilized to establish point patterns of these data to detect potential clusters of communities, and the statistically detected clusters can be examined using high resolution maps of local industries and roadways or remote sensing imagery like nightlight 
data as surrogates for population density with a higher level of granularity. Analyzing clusters in the context of the local geographical and industrial dynamics facilitates better insight into the hyperlocal structure of these hidden communities and facilitates future hotspot identification. The focus of specific populations for small area estimation models were selected

based on where there were enough data to use as a starting place. Specific subnational areas were selected to coincide most closely with the PEPFAR-defined organizational units.

A few examples of the focus, data employed, methods, and findings of small area estimation exercises for LINKAGES and GF priority countries follow.

\section{Côte d'Ivoire: Bayesian estimation of MSM population size}

\section{Data}

Information on MSM population size was available from a number of sources at five departments (a primary administrative unit) of Côte d'Ivoire: Abidjan, Agboville, Bouake, Gagnoa, and Yamoussoukro. Counts of MSM were taken from program data for NGO membership, service provision, and social event attendance.

\section{Method}

Available data were used in a Bayesian regression model to produce estimates of the numbers of MSM in areas of Côte d'Ivoire prioritized in the HIV response (PEPFAR Organizational Units). The hierarchical model imputes missing covariates, including HIV prevalence, using geo-spatial information and allows for proper uncertainty quantification leading to confidence bounds for predicted MSM PSEs. This process provided PSEs where there are no empirical data, to guide the prioritization of further collection of empirical data on MSM and inform evidence-based scaling of HIV prevention and treatment programs for MSM across Côte d'Ivoire.

\section{Results}

In terms of absolute numbers, small area estimation found that Abidjan, though it has one of the lowest proportions of MSM in the general population, has by far the highest predicted MSM population size, given the large size of the Abidjan population. For areas without direct estimates, the predicted MSM population percentage typically varied between as low as 0.5 percent to around 10 percent. The highest MSM percentages were predicted to be in Katiola, Kouassikouassikro, and Bettie. However, these areas also had the widest credible intervals indicating the large uncertainties associated with the predictions. Wider confidence intervals mean less confidence about predicted percentages at the higher end of the range.

Additional information is provided in Appendix D, in a published manuscript of this work. 


\section{Malawi: Female sex worker size estimation at the district level}

\section{Data}

Two data sources for FSW population size at the subnational level were found for Malawi: The Priorities for Local AIDS Control Efforts (PLACE) report and the 2013-2014 Malawi Biological and Behavioral Surveillance Survey (BBSS) Report. For auxiliary data, the 2015-2016 Malawi Demographic and Health Survey (DHS) was used.

\section{Method}

In considering the small area estimation approach for this particular set of data, there were 14 districts with direct size estimates and 936 possible auxiliary data candidates from the DHS. In order to determine which of the 936 DHS variables would serve as predictors, pairwise correlations between each predictor and the size estimates were calculated. The top 10 predictors were selected based on the variables that had the smallest p-values, and these predictors were further condensed into three sets of predictors. Those variables that had higher than 0.9 correlation with others were removed from consideration. To complete the small area estimation, in this instance a mixed effects model was built with fixed effects being either the directly combined predictors or the factors and the random effects being the data source.

\section{Results}

During this activity, PLACE II data became available. Combining PLACE I and II data together as the PLACE data, the model described above was re-run. Because of this newly released data, it was possible to compare originally extrapolated estimates to the newly collected data. This exercise served as a validation tool. Based on the validation, we saw that the factor model produced more stable and shorter prediction intervals in districts without any observed data. For many of the districts that we were able to validate based on PLACE II data, the estimates were overlapping. In three districts, Mwanza, Nkhotakota, and Salima, the PLACE II numbers were higher than the predicted ones.

\section{Eswatini: Bayesian multi-region population size estimation using incomplete and misaligned capture-recapture data}

\section{Data}

Eswatini is divided into four administrative regions: Hhohho, Lubombo, Manzini, and Shiselweni. The goal of the analysis was to estimate, with proper uncertainty quantification, the number of MSM and FSWs in each of these four regions. Separate surveys were conducted for both these populations in 2014 using the PLACE method. A modified version of the PLACE method was used to characterize venues where MSM and FSWs meet new potential sexual partners. Data from additional sources made use of a multiplier method (unique object, coupon distribution at social events, service) (Family Life Association of Swaziland ${ }^{1}$ online) These additional data sources included survey data and a list of the total unique objects distributed, a list of the total HIV service coupons handed out, a total number of MSM attending a Rainbow Night social event, and a list of the total number of individuals who sought services from Family Life Association of Swaziland. The multiplier methods make use of the survey data and these different lists to get estimates of direct population size.

${ }^{1}$ Swaziland officially renamed itself Eswatini in 2018. 


\section{Method}

To characterize the distributions of marginalized communities in Eswatini, the scenario when multiple data sources are available at each region for the multiplier method was considered. Current practices in such circumstances produce multiple PSEs at each region and often ignore the correlation among these estimates. We recast the multiple multiplier data problem into a multiple capture-recapture problem with incomplete data and proposed a fully modelbased approach for size estimation using multiple capture-recapture data with an arbitrary pattern of incompleteness. A data augmentation scheme was used that allowed for modeling the correlations in the data and producing a unified estimate of population size per region. A hierarchical Bayesian model tied together the models for multiple regions and allowed for borrowing strength across the regions. Eswatini data misalignment where counts from some of the data sources are not available for each region but are available as an aggregate over a few regions was also encountered. A solution to the general misalignment problem was to consider data-source-specific patterns of misalignment. Simulation studies demonstrated the accurate inferential capabilities of this model. This approach was then used to produce uncertaintyquantified PSEs of key populations in Eswatini.

\section{Results}

Uncertainty quantified size estimates of MSM and FSWs for all four regions of Eswatini are listed in tables 4 and 5.

Table 4 Region specific size estimates of FSWs in the age group of 15-49 years

\begin{tabular}{|lccc|}
\hline Region & $\begin{array}{c}\text { Female } \\
\text { 15-49 population }\end{array}$ & FSW size estimate & FSW proportion \\
\hline Hhohho & 84,784 & $6,415(5,464-7,493)$ & $7.6 \%(6.4-8.8 \%)$ \\
Manzini & 100,360 & $2,114(1,872-2,377)$ & $2.1 \%(1.9-2.4 \%)$ \\
Shiselweni & 48,391 & $2,052(1,568-2,722)$ & $4.2 \%(3.2-5.6 \%)$ \\
Lubombo & 52,762 & $4,059(2,045-8,328)$ & $7.7 \%(3.9-16.0 \%)$ \\
\hline
\end{tabular}

Table 5 Region specific size estimates of MSM in the age group of 15-49years

\begin{tabular}{|lccc|}
\hline Region & $\begin{array}{c}\text { Male } \\
\text { 15-49 population }\end{array}$ & MSM size estimate & MSM proportion \\
\hline Hhohho & 85,918 & $3,465(2,888-4,176)$ & $4.0 \%(3.4-4.7 \%)$ \\
Manzini & 95,720 & $2,650(2,281-3,068)$ & $2.8 \%(2.4-3.2 \%)$ \\
Shiselweni & 15,216 & $1,869(1,544-2,238)$ & $4.1 \%(3.4-4.9 \%)$ \\
Lubombo & 52,519 & $2,015(1,451-2,764)$ & $3.8 \%(2.8-5.3 \%)$ \\
\hline
\end{tabular}




\section{OBJECTIVE: DYNAMIC TRANSMISSION MODELING}

Rigorously evaluate, through systematic reviews and generic modeling, the contribution of FSWs and their clients and MSM in diverse HIV epidemic contexts across sub-Saharan Africa.

\section{FSWs and clients of FSWs}

An independent systematic review was conducted for this activity. Published (Medline, EMBASE, PsychInfo, Scopus) and grey literature were systematically reviewed to empirically estimate the population size and HIV prevalence/incidence of FSWs and/or clients, by SSA country/region and year of data collection (2002-2013). Static model-based estimates of incidence rate ratios of sex work from the UNAIDS HIV Modes of Transmission (MOT) models were reviewed and we updated a systematic search of dynamic model estimates looking at the potential population impact of sex work interventions. The odds ratio (OR) of prevalent HIV in FSWs/clients compared to adult females and non-clients was estimated using national HIV prevalence estimates by year of data collection, the classic population attributable fraction (PAF) of sex work on prevalent HIV in males and females by country; and used dynamic models of HIV transmission in three settings to explore the utility of the classic PAF when appraising the contribution of sex work to HIV epidemics.

This review found that the median size of FSW and client populations were 2 percent (range, 0.25-11.5 percent, 35 countries) and 3 percent (range, 0.025-30.0 percent, 36 countries) respectively. The pooled HIV prevalence in FSWs and clients was 28 percent (95 percent Cl: $24.6-$ $31.9, \mathrm{~N}=79,34$ countries) and 6 percent (95 percent $\mathrm{Cl}$ : 4.3-8.9 percent, $\mathrm{N}=39,27$ countries) respectively. The pooled OR among FSWs was 9.7 (95 percent Cl: 7.9-11.9), varying by region and decreasing over time: 13.4, 9.8, and 5.6 in 2002-2005, 2006-2009, and 2010-2013 respectively. The pooled OR among clients was 2.0 (95 percent Cl: 1.4-2.8). The MOT-derived incidence rate ratios for FSWs and clients ranged from 2.3 to 17.4 , and 1.1 to 26.3 , respectively $(\mathrm{N}=13)$. The classic PAF of sex work on prevalent HIV infections in females and males ranged from 0.4 percent to 71 percent and 0 to 88 percent respectively, while relative HIV expenditure on sex work programs was 0 to 3 percent (17 countries). Dynamic model analyses from Benin, Burkina Faso, and Kenya showed that the classic PAF considerably underestimates the medium- to longterm contribution of sex work to HIV epidemics. Ten dynamic models identified from the modeling review suggest that across a range of interventions, focused FSW programs could avert up to 85 percent of new HIV infections over 15 years, and reduce HIV incidence by up to 35 percent over 10 years in the wider community. The results of the FSW and client reviews are in Appendix E.

\section{MSM}

Similarly, for MSM we conducted an independent systematic review and meta-analysis according to Preferred Reporting Items for Systematic Reviews and Meta-Analyses (PRISMA) and Metaanalysis of Observational Studies in Epidemiology guidelines. The study protocol is registered in PROSPERO (ID: CRD42016039518; available from http://www.crd.york.ac.uk/PROSPERO/ display_record.php?ID=CRD42016039518). Primary outcomes in relation to the 90-90-90 targets included: (1) proportion HIV diagnosed and aware (self-reported awareness of their HIVpositive status) among individuals with a laboratory confirmed HIV-positive test; proportion of ART 
uptake (ever initiated ART; currently on ART) among individuals living with HIV, or diagnosed with HIV and aware of their HIV status; and (3) proportion achieving viral load suppression (defined as viral load suppression at the most recent follow-up visit) among individuals taking ART. Secondary outcomes included all other elements of the HIV care continuum: linkage to HIV care within one year of diagnosis; CD4 at diagnosis; viral load at diagnosis; retention in care; loss to care; ART attrition (discontinuation or death); ART adherence; and time from HIV diagnosis to HIV care, ART initiation, and viral load suppression.

For each outcome, proportions or rates (numerators, denominators, adjusted point estimates, and confidence intervals) or time-based events (unit of time, mean, median, standard deviation, range, and interquartile range) were extracted. Adjusted (in studies using RDS, weighted, or probabilistic sampling strategies) and crude estimates were extracted where available.

Briefly, we identified 88 studies (59 unique populations) which met inclusion criteria and provided data on 8,902 MSM living with HIV in 20 SSA countries. Across 53 unique study populations in 18 SSA countries ( 3 studies conducted in multiple countries) from 58 studies, the pooled prevalence of HIV diagnosis and awareness among MSM living with HIV in SSA was 27 percent (95 percent Cl: 22.0-33.1, $12=95.2$ percent, $\mathrm{Ns}=54, \mathrm{~Np}=43)$. In West/Central, East, and Southern Africa, the pooled proportion of diagnosed and aware among MSM living with HIV was 35 percent (95 percent $\mathrm{Cl} 28.0-42.6, \mathrm{I} 2=94.8$ percent, $\mathrm{Ns}=26, \mathrm{~Np}=21$ ); 23 percent (95 percent $\mathrm{Cl} 8.9-48.7$, $\mathrm{I} 2=96.7$ percent, $\mathrm{Ns}=\mathrm{Np}=7$ ); and 28 percent $(95$ percent $\mathrm{Cl} 18.3-40.7, \mathrm{I} 2=95.4$ percent, $\mathrm{Ns}$ $=25, \mathrm{~Np}=18$ ), respectively. The proportion of MSM diagnosed with HIV and currently on ART at the time of the study was 44 percent $(95$ percent $\mathrm{Cl} 33.0-55.8, \mathrm{I} 2=97.8$ percent, $\mathrm{Ns}=31, \mathrm{~Np}=$ 28 ) and MSM diagnosed with HIV who were ever initiated on ART was 55 percent (95 percent $\mathrm{Cl}$ 35.1-73.0, $12=90.8$ percent, $\mathrm{Ns}=12, \mathrm{~Np}=11$ ). Among MSM on ART, 45 percent (95 percent $\mathrm{Cl} 24.1-68.4, \mathrm{I} 2=96.2$ percent, $\mathrm{Ns}=9, \mathrm{~Np}=7$ ) were virally suppressed. The largest source of variability was the type of setting in which data were collected. The cascade was consistently better when data were drawn from research clinics or program clinics, with the lowest level of diagnoses, ART use, and viral suppression reported via population-based surveys. The systematic review and analysis results are shown in Appendix F. 


\section{CONCLUSION}

Because of this activity, a series of novel and innovative resources are now available for local and global stakeholders. A global systematic review was completed of all available data published between 2006 and 2019 characterizing the burden of HIV, the HIV treatment cascade, key prevention indicators, PSEs, and select structural determinants of HIV among key populations. This valuable resource has already been used by organizations such as UNAIDS, USAID headquarters, the World Health Organization, FHI360, and Population Council, among others. There has also been a request to integrate this comprehensive and important review into the KP Atlas, a collaborative effort with USAID that is now underway. Ultimately, the KP Atlas, with the global systematic review incorporated, will provide key stakeholders with a user-friendly data platform and website that displays HIV status for key populations in more than 150 countries.

Quality assessments were conducted on available data for 30 priority countries based on an adapted assessment. The results of the quality assessments have been shared with participants at capacity strengthening workshops, are housed in a REDcap data repository along with all data records, and can be accessed upon request. These assessments will eventually be available through the KP Atlas. An assessment of the utilization of epidemiologic data was also conducted and showed, for example, that only a small proportion of size estimation studies are being used to inform programming within country governments and their funding and implementation partners, including USAID and the Global Fund. This finding underscores the importance of improving methods and tools to ensure the effective uptake of epidemiological data, including size estimation data.

Capacity building workshops were conducted in Cameroon, Cote d'Ivoire, Eswatini, Malawi, South Africa, and Zambia and aimed to highlight existing data and promote better understanding of epidemiologic data and its application to policy development. In several instances, small area size estimation and dynamic modeling were conducted and shared in capacity-strengthening workshops to further strengthen the utility of available data. Overall, workshop attendees and stakeholders expressed strong interest in improving the quality of future data collection, filling data gaps, and further examining data they have collected according to the applied quality assessment tool. At the completion of each workshop, at the request of local stakeholders and in agreement with USAID headquarters, USAID country offices, and GF, ongoing technical assistance has been provided to in-country stakeholders. Specific technical assistance aims to support improved data to inform responses, improved methods to characterize needs, and improved decision-making in response to different levels of quality of data.

The work reported here, including the results of data synthesis and dynamic modeling, have also informed COP 2019 activities at both a headquarters and local level. COP stakeholders were made aware of primary vs secondary sources of available data and subsequent implications around quality, empowering them to make decisions on which data to utilize during engagement. Specific examples of engagement with the COP process include using the Global.HIV data repository to fill a request from USAID headquarters for data reports on all available global key 
population data on HIV prevalence and treatment cascade indicators. In another example, at the local level, modeling was conducted for USAID South Africa to predict the impact of decreased condom use in sexual partnerships due to prevention funding cuts on overall prevalence and incidence and estimates of transmissible PAF were developed with 1, 5, 10, and 20 year timelines estimating infections among sex workers, clients, MSM, and the wider population for the COP 19 South Africa meetings.

The overarching goal of this activity was to increase the use of high-quality data to inform both the scale and content of HIV prevention and treatment programs for key populations in high impact GF and USAID countries. The development of a comprehensive global systematic review which will be widely accessible to stakeholders, as well as the methods to conduct quality assessments and to strengthen data utilization that this project supported, have added to both the depth and breadth of data available as well as the tools that can be used for evaluating and using data for judicious planning and resource allocation. Moving forward, the lessons learned suggest the need for an activity like this to be scaled up to all countries to ensure an increasingly efficient, evidence-based, and human rights affirming HIV response. 


\section{REFERENCES}

Covidence. Better systematic review management: A Cochrane Techology Platform [Available from: https://www.covidence.org/home.

FLAS. Family Life Association of Swaziland Manzini, Swaziland [Available from: http://www.flas.org.sz/

Moher, D. et al. 2009. "Preferred reporting items for systematic reviews and meta-analyses: the PRISMA Statement," Open Medicine 3(3): e123-30.

NHLBI. Study Quality Assessment Tools Bethesda, MD [Available from: https://www.nhlbi.nih.gov/ health-topics/study-quality-assessment-tools.

NICHSR. HSRProj (Health Services Research Projects in Progress) Bethesda, MD [Available from: https://hsrproject.nlm.nih.gov/.

USAID. Development Experience Clearinghouse [Available from: https://dec.usaid.gov/dec/home/ Default.aspx].

\section{PEER-REVIEWED PAPERS PUBLISHED TO DATE}

Datta, A. et al. 2019. "Bayesian estimation of MSM population in Cote d'Ivoire," Statistics and Public Policy 6(1): 1-13. doi: 10.1080/2330443X.2018.1546634

Edwards, J.K. et al. "Estimating sizes of key populations at the national level: considerations for study design and analysis," Epidemiology 29(6): 795-803. doi: 10.1097/EDE.0000000000000906

Rao, A. et al. 2018. "HIV-related data among key populations to inform evidence-based responses: protocol of a systematic review," Systematic Reviews 7(1): 220. doi: 10.1186/s13643-018-0894-3

Check back for more! 


\section{APPENDICES}

Appendices are available on the Project SOAR website (projsoar.org).

Appendix A: Key Populations Atlas Advancement Plan

Appendix B: Example data report generated by Global.HIV data repository

Appendix C: Quality Assessment Tool and Scoring

Appendix D: Small area estimation publication

Appendix E: Results of systematic review among FSW and clients

Appendix F: Results of systematic review among MSM

Appendix G: Malawi Workshop Final Report

Appendix H: Eswatini Workshop Final Report

Appendix I: Zambia Workshop Final Report

Appendix J: South Africa Workshop Final Report 
Project SOAR

Population Council 4301 Connecticut Ave, NW,

Suite 280

Washington, D.C. 20008 USA

Tel: +1 2022379400

Fax: +1 2022378410

Ideas. Evidence. Impact.

projsoar.org 